\title{
Coleman-Weinberg Potential In Good Agreement With WMAP
}

\author{
Q. Shaft* and V. N. Şenoğudt \\ Bartol Research Institute, Department of Physics and Astronomy, \\ University of Delaware, Newark, DE 19716, USA
}

\begin{abstract}
We briefly summarize and update a class of inflationary models from the early eighties based on a quartic (Coleman-Weinberg) potential for a gauge singlet scalar (inflaton) field. For vacuum energy scales comparable to the grand unification scale, the scalar spectral index $n_{s} \simeq 0.94-0.97$, in very good agreement with the WMAP three year results. The tensor to scalar ratio $r \lesssim 0.14$ while $\alpha \equiv \mathrm{d} n_{s} / \mathrm{d} \ln k$ is $\simeq-10^{-3}$. An $S O(10)$ version naturally explains the observed baryon asymmetry via non-thermal leptogenesis.

PACS numbers: $98.80 . \mathrm{Cq}$
\end{abstract}

An inflationary scenario [1, 2] may be termed successful if it satisfies the following criteria:

1) The total number of $e$-folds $\mathrm{N}$ during inflation is large enough to resolve the horizon and flatness problems. Thus, $N \gtrsim 50-60$, but it can be somewhat smaller for low scale inflation.

2) The predictions are consistent with observations of the microwave background and large scale structure formation. In particular, the predictions for $n_{s}, r$ and $\alpha$ should be consistent with the most recent WMAP results [3] (see also [4] for a brief survey of models).

3) Satisfactory resolution of the monopole problem in grand unified theories (GUTs) is achieved.

4) Explanation of the origin of the observed baryon asymmetry is provided.

In this report we review a class of inflation models which appeared in the early eighties in the framework of non-supersymmetric GUTs and employed a GUT singlet scalar field $\phi$ [5, 6, 7]. These (Shafi-Vilenkin) models satisfy, as we will see, the above criteria and are

*Electronic address: shafi@bartol.udel.edu

†Electronic address: nefer@udel.edu 
based on a Coleman-Weinberg $(\mathrm{CW})$ potential [8]

$$
V(\phi)=V_{0}+A \phi^{4}\left[\ln \left(\frac{\phi^{2}}{M_{*}^{2}}\right)+C\right]
$$

where, following [5] the renormalization mass $M_{*}=10^{18} \mathrm{GeV}$ and $V_{0}^{1 / 4}$ will specify the vacuum energy. The value of $C$ is fixed to cancel the cosmological constant at the minimum. It is convenient to choose a physically equivalent parametrization for $V(\phi)$ [9, 10], namely

$$
V(\phi)=A \phi^{4}\left[\ln \left(\frac{\phi}{M}\right)-\frac{1}{4}\right]+\frac{A M^{4}}{4},
$$

where $M$ denotes the $\phi \mathrm{VEV}$ at the minimum. Note that $V(\phi=M)=0$, and the vacuum energy density at the origin is given by $V_{0}=A M^{4} / 4$.

The potential above is typical for the new inflation scenario [2], where inflation takes place near the maximum. However, as we discuss below, depending on the value of $V_{0}$, the inflaton can have small or large values compared to the Planck scale during observable inflation. In the latter case observable inflation takes place near the minimum and the model mimics chaotic inflation [11].

The original new inflation models attempted to explain the initial value of the inflaton through high-temperature corrections to the potential. This mechanism does not work unless the inflaton is somewhat small compared to the Planck scale at the Planck epoch 10]. However, the initial value of the inflaton could also be suppressed by a pre-inflationary phase. Here we will simply assume that the initial value of the inflaton is sufficiently small to allow enough $e$-folds.

The slow-roll parameters may be defined as [12]

$$
\epsilon=\frac{1}{2}\left(\frac{V^{\prime}}{V}\right)^{2}, \quad \eta=\left(\frac{V^{\prime \prime}}{V}\right), \quad \xi^{2}=\left(\frac{V^{\prime} V^{\prime \prime \prime}}{V^{2}}\right) .
$$

(Here and below we use units $m_{P}=1$, where $m_{P} \simeq 2.4 \times 10^{18} \mathrm{GeV}$ is the reduced Planck mass.) The slow-roll approximation is valid if the slow-roll conditions $\epsilon \ll 1$ and $\eta \ll 1$ hold. In this case the spectral index $n_{\mathrm{s}}$, the tensor-to-scalar ratio $r$ and the running of the spectral index $\alpha \equiv \mathrm{d} n_{\mathrm{s}} / \mathrm{d} \ln k$ are given by

$$
\begin{aligned}
n_{\mathrm{s}} & \simeq 1-6 \epsilon+2 \eta \\
r & \simeq 16 \epsilon \\
\alpha & \simeq 16 \epsilon \eta-24 \epsilon^{2}-2 \xi^{2} .
\end{aligned}
$$


The number of $e$-folds after the comoving scale $l_{0}=2 \pi / k_{0}$ has crossed the horizon is given by

$$
N_{0}=\frac{1}{2} \int_{\phi_{e}}^{\phi_{0}} \frac{H(\phi) \mathrm{d} \phi}{H^{\prime}(\phi)}
$$

where $\phi_{0}$ is the value of the field when the scale corresponding to $k_{0}$ exits the horizon and $\phi_{e}$ is the value of the field at the end of inflation. This value is given by the condition $2\left(H^{\prime}(\phi) / H(\phi)\right)^{2}=1$, which can be calculated from the Hamilton-Jacobi equation [13]

$$
\left[H^{\prime}(\phi)\right]^{2}-\frac{3}{2} H^{2}(\phi)=-\frac{1}{2} V(\phi) .
$$

The amplitude of the curvature perturbation $\mathcal{P}_{\mathcal{R}}^{1 / 2}$ is given by

$$
\mathcal{P}_{\mathcal{R}}^{1 / 2}=\frac{1}{2 \sqrt{3} \pi} \frac{V^{3 / 2}}{\left|V^{\prime}\right|}
$$

To calculate the magnitude of $A$ and the inflationary parameters, we use these standard equations above. We also include the first order corrections in the slow roll expansion for $\mathcal{P}_{\mathcal{R}}^{1 / 2}$ and the spectral index $n_{s}$ [14]. ${ }^{1}$ The WMAP value for $\mathcal{P}_{\mathcal{R}}^{1 / 2}$ is $4.86 \times 10^{-5}$ for $k_{0}=$ $0.002 \mathrm{Mpc}^{-1} . N_{0}$ corresponding to the same scale is $\simeq 53+(2 / 3) \ln \left(V\left(\phi_{0}\right)^{1 / 4} / 10^{15} \mathrm{GeV}\right)+$ $(1 / 3) \ln \left(\mathrm{T}_{\mathrm{r}} / 10^{9} \mathrm{GeV}\right)$. (The expression for $N_{0}$ assumes a standard thermal history [15]. See 16] for reviews.) We assume reheating is efficient enough such that $T_{r}=m_{\phi}$, where the mass of the inflaton $m_{\phi}=2 \sqrt{A} M$. In practice, we expect $T_{r}$ to be somewhat below $m_{\phi}[5]$.

In Table I and Fig. 1 we display the predictions for $n_{s}, \alpha$ and $r$, with the vacuum energy scale $V_{0}^{1 / 4}$ varying from $10^{13} \mathrm{GeV}$ to $10^{17} \mathrm{GeV}$. The parameters have a slight dependence on the reheat temperature, as can be seen from the expression for $N_{0}$. As an example, if we assume instant reheating $\left(T_{r} \simeq V\left(\phi_{0}\right)^{1 / 4}\right), n_{s}$ would increase to 0.941 and 0.943 for $V_{0}^{1 / 4}=10^{13} \mathrm{GeV}$ and $V_{0}^{1 / 4}=10^{15} \mathrm{GeV}$ respectively.

For $V_{0}^{1 / 4} \lesssim 10^{16} \mathrm{GeV}$, the inflaton field remains smaller than the Planck scale, and the inflationary parameters are similar to those for new inflation models with $V=V_{0}\left(1-(\phi / \mu)^{4}\right)$ : $n_{s} \simeq 1-\left(3 / N_{0}\right), \alpha \simeq\left(n_{s}-1\right) / N_{0}$. As the vacuum energy is lowered, $N_{0}$ becomes smaller and $n_{s}$ deviates further from unity. However, $n_{s}$ remains within $2 \sigma$ of the WMAP best fit

\footnotetext{
${ }^{1}$ The fractional error in $\mathcal{P}_{\mathcal{R}}^{1 / 2}$ from the slow roll approximation is of order $\epsilon$ and $\eta$ (assuming these parameters remain $\ll 1$ ). This leads to an error in $n_{s}$ of order $\xi^{2}$, which is $\sim 10^{-3}$ in the present model. Comparing to the WMAP errors, this precision seems quite adequate. However, in anticipation of the Planck mission, it may be desirable to consider improvements.
} 
TABLE I: The inflationary parameters for the Shafi-Vilenkin model with the potential in Eq. (2) $\left(m_{P}=1\right)$

\begin{tabular}{rrrrrrrrr}
\hline \hline$V_{0}^{1 / 4}(\mathrm{GeV})$ & $A\left(10^{-14}\right)$ & $\mathrm{M}$ & $\phi_{e}$ & $\phi_{0}$ & $V\left(\phi_{0}\right)^{1 / 4}(\mathrm{GeV})$ & $n_{s}$ & $\alpha\left(-10^{-3}\right)$ & $r$ \\
\hline $10^{13}$ & 1.0 & 0.018 & 0.010 & $3.0 \times 10^{-6}$ & $\approx V_{0}^{1 / 4}$ & 0.938 & 1.4 & $9 \times 10^{-15}$ \\
\hline $5 \times 10^{13}$ & 1.2 & 0.088 & 0.050 & $7.5 \times 10^{-5}$ & $\approx V_{0}^{1 / 4}$ & 0.940 & 1.3 & $5 \times 10^{-12}$ \\
\hline $10^{14}$ & 1.3 & 0.17 & 0.10 & $3.0 \times 10^{-4}$ & $\approx V_{0}^{1 / 4}$ & 0.940 & 1.2 & $9 \times 10^{-11}$ \\
\hline $5 \times 10^{14}$ & 1.9 & 0.79 & 0.51 & $7.5 \times 10^{-3}$ & $\approx V_{0}^{1 / 4}$ & 0.941 & 1.2 & $5 \times 10^{-8}$ \\
\hline $10^{15}$ & 2.3 & 1.5 & 1.1 & 0.030 & $\approx V_{0}^{1 / 4}$ & 0.941 & 1.2 & $9 \times 10^{-7}$ \\
\hline $5 \times 10^{15}$ & 4.8 & 6.2 & 5.1 & 0.71 & $\approx V_{0}^{1 / 4}$ & 0.942 & 1.0 & $5 \times 10^{-4}$ \\
\hline $10^{16}$ & 5.2 & 12 & 10 & 3.2 & $9.9 \times 10^{15}$ & 0.952 & 1.0 & $8 \times 10^{-3}$ \\
\hline $2 \times 10^{16}$ & 1.1 & 36 & 35 & 23 & $1.7 \times 10^{16}$ & 0.966 & 0.6 & 0.07 \\
\hline $3 \times 10^{16}$ & .17 & 86 & 85 & 72 & $1.9 \times 10^{16}$ & 0.967 & 0.6 & 0.11 \\
\hline $10^{17}$ & .001 & 1035 & 1034 & 1020 & $2.0 \times 10^{16}$ & 0.966 & 0.6 & 0.14 \\
\hline \hline
\end{tabular}

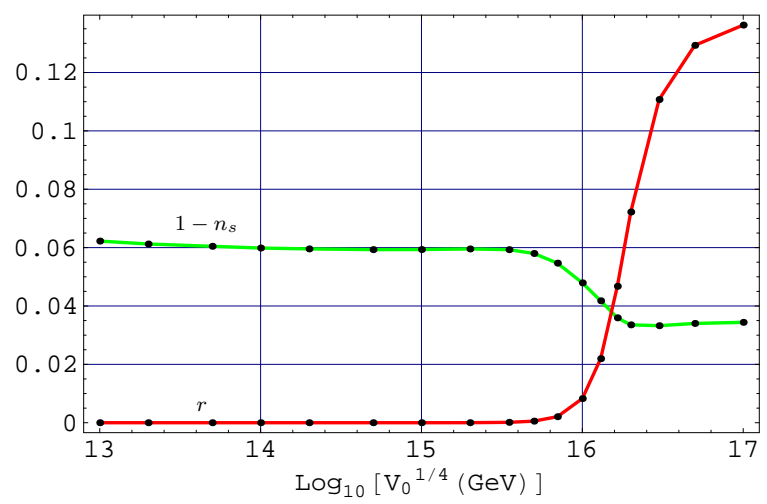

FIG. 1: $1-n_{s}$ and $r$ vs. $\log \left[V_{0}^{1 / 4}(\mathrm{GeV})\right]$ for the potential in Eq. (2) .

value (for negligible $r$ ) $0.951_{-0.019}^{0.015}$ [3] even for $V_{0}^{1 / 4}$ as low as $10^{5} \mathrm{GeV}$. Inflation with $\mathrm{CW}$ potential at low scales is discussed in Ref. 17].

For $V_{0}^{1 / 4} \gtrsim 10^{16} \mathrm{GeV}$, the inflaton is larger than the Planck scale during observable inflation. Observable inflation then occurs closer to the minimum where the potential is effectively $V=(1 / 2) m_{\phi}^{2} \Delta \phi^{2}, \Delta \phi=M-\phi$ denoting the deviation of the field from the minimum. This well-known monomial model [11] predicts $m_{\phi} \simeq 2 \times 10^{13} \mathrm{GeV}$ and $\Delta \phi_{0} \simeq$ $2 \sqrt{N_{0}}$, corresponding to $V\left(\phi_{0}\right) \simeq\left(2 \times 10^{16} \mathrm{GeV}\right)^{4}$. For the $\phi^{2}$ potential to be a good approximation, $V_{0}$ must be greater than this value. Then the inflationary parameters no longer depend on $V_{0}$ and approach the predictions for the $\phi^{2}$ potential.

The tensor to scalar ratio $r$ versus the spectral index $n_{s}$ is displayed in Fig. 2. The 


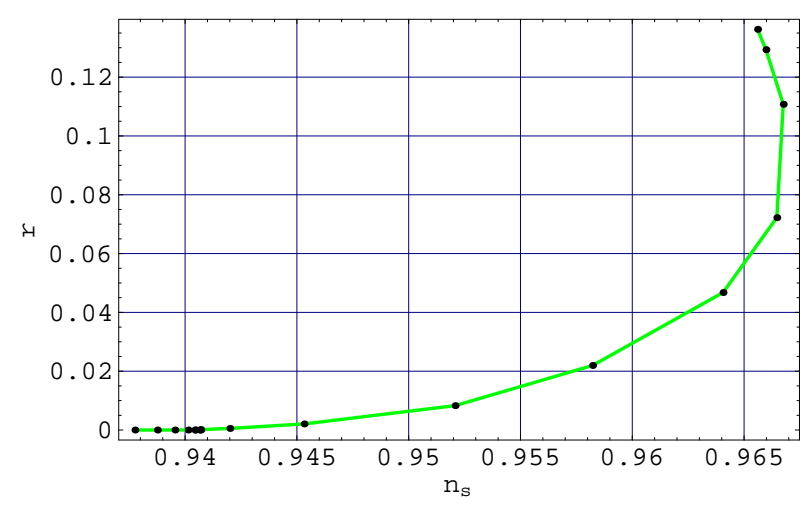

FIG. 2: $r$ vs. $n_{s}$ for the potential in Eq. (2).

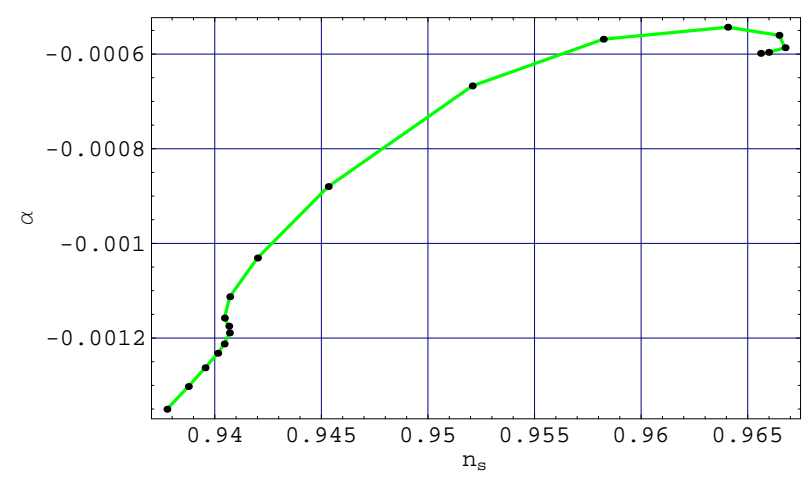

FIG. 3: $\alpha$ vs. $n_{s}$ for the potential in Eq. (2) .

values are in very good agreement with the recent WMAP results (see Fig. 14 of Ref. [3] ). The running of the spectral index is negligible, as in most inflation models (Fig. 31). Note that the WMAP data favor a large running spectral index. This is an important result if confirmed but currently has little statistical significance.

In the context of non-supersymmetric GUTs, $V_{0}^{1 / 4}$ is related to the unification scale, and is typically a factor of 3-4 smaller than the superheavy gauge boson masses due to the loop factor in the CW potential. The unification scale for non-supersymmetric GUTs is typically $10^{14}-10^{15} \mathrm{GeV}$, although it is possible to have higher scales, for instance associating inflation with $S O(10)$ breaking via $S U(5)$.

The reader may worry about proton decay with gauge boson masses of order $10^{14}-10^{15}$ $\mathrm{GeV}$. In the $S U(5)$ model [18], in particular, a two-loop renormalization group analysis of the standard model gauge couplings yields masses for the superheavy gauge bosons of order $1 \times 10^{14}-5 \times 10^{14} \mathrm{GeV}[19]$. This is consistent with the SuperK proton lifetime limits [20], provided one assumes strong flavor suppression of the relevant dimension six gauge mediated proton decay coefficients. If no suppression is assumed the gauge boson masses should have 
masses close to $10^{15} \mathrm{GeV}$ or higher [21].

For the Shafi-Vilenkin model in $S U(5)$, the tree level scalar potential contains the term $(1 / 2) \lambda \phi^{2} \operatorname{Tr} \Phi^{2}$ with $\Phi$ being the Higgs adjoint, and $A \sim 1.5 \times 10^{-2} \lambda^{2}[\underline{5}$, 10]. Inflation requires $A \sim 10^{-14}$, corresponding to $\lambda \sim 10^{-6}$.

This model has been extended to $S O(10)$ in Ref. [7]. The breaking of $S O(10)$ to the standard model proceeds, for example, via the subgroup $G_{422}=S U(4)_{c} \times S U(2)_{L} \times S U(2)_{R}$ 22]. A renormalization group analysis shows that the symmetry breaking scale for $S O(10)$ is of order $10^{15} \mathrm{GeV}$, while $G_{422}$ breaks at an intermediate scale $M_{I} \sim 10^{12} \mathrm{GeV}$ [23]. (This is intriguingly close to the scale needed to resolve the strong CP problem and produce cold dark matter axions.) The predictions for $n_{s}, \alpha$ and $r$ are essentially identical to the $\mathrm{SU}(5)$ case. There is one amusing consequence though which may be worth mentioning here. The monopoles associated with the breaking of $S O(10)$ to $G_{422}$ are inflated away. However, the breaking of $G_{422}$ to the SM gauge symmetry yields doubly charged monopoles [24], whose mass is of order $10^{13} \mathrm{GeV}$. These may be present in our galaxy at a flux level of $10^{-16} \mathrm{~cm}^{-2}$ $\mathrm{s}^{-1} \mathrm{Sr}^{-1}$ [7].

As stated earlier, before an inflationary model can be deemed successful, it must contain a mechanism for generating the observed baryon asymmetry in the universe. In the $S U(5)$ case the color higgs triplets produced by the inflaton decay can generate the baryon asymmetry, provided the higgs sector of the model has the required amount of CP violation [5].

The discovery of neutrino oscillations requires that we introduce SU(5) singlet right handed neutrinos, presumably three of them, to implement the seesaw mechanism and generate the desired masses for the light neutrinos. In this case it is natural to generate the observed baryon asymmetry via leptogenesis [25] (for non-thermal leptogenesis see Ref. [26]) by introducing the couplings $N_{i} N_{j} \phi^{2} / m_{P}$, where $N_{i}(\mathrm{i}=1,2,3)$ denote the right handed neutrinos, and the renormalizable coupling to $\phi$ is absent because of the assumed discrete symmetry. By suitably adjusting the Yukawa coefficients one can arrange that the $\phi$ field decays into the right handed neutrinos. Note that the presence of the above Yukawa couplings then allows one to make the color triplets heavier, of order $10^{14} \mathrm{GeV}$, thereby avoiding any potential conflict with proton decay. In the $S O(10)$ model, leptogenesis is almost automatic [7].

Finally, it is worth noting that new inflation models have also been considered in the framework of supersymmetric GUTs, taking account of supergravity corrections. In Ref. 
27], for instance, it is shown that the spectral index $n_{s}$ is less than 0.98 , with values between 0.94 and 0.96 plausible. Furthermore, reheat temperatures as low as $10^{4}-10^{6} \mathrm{GeV}$ can be realized to satisfy the gravitino constraint. In these models the tensor to scalar ratio $r$ is tiny, of order $10^{-3}$ or less, and $\alpha \sim-10^{-3}$.

To summarize, we have briefly reviewed and updated a class of realistic inflation models based on a quartic CW potential for a gauge singlet inflaton field. We find very good agreement between the model predictions and the three year WMAP data. An interesting feature is the observation that if the vacuum energy that drives inflation exceeds $10^{16} \mathrm{GeV}$, the inflaton during observable inflation exceeds the Planck mass in value. As a consequence, there is transition from new to chaotic inflation (or more precisely the monomial potential model), and the scalar spectral index and $r$ acquire limiting values of 0.966 and 0.14 respectively.

\section{Acknowledgements}

This work is partially supported by the US Department of Energy under contract number DE-FG02-91ER40626 (Q.S. and V.N.S.), and by a University of Delaware graduate fellowship (V.N.S.).

[1] A. H. Guth, Phys. Rev. D23, 347 (1981).

[2] A. D. Linde, Phys. Lett. B108, 389 (1982); A. Albrecht and P. J. Steinhardt, Phys. Rev. Lett. 48, 1220 (1982).

[3] D. N. Spergel et al. (2006), astro-ph/0603449.

[4] L. Alabidi and D. H. Lyth, (2006), astro-ph/0603539.

[5] Q. Shafi and A. Vilenkin, Phys. Rev. Lett. 52, 691 (1984).

[6] S.-Y. Pi, Phys. Rev. Lett. 52, 1725 (1984); Q. Shafi and A. Vilenkin, Phys. Rev. D29, 1870 (1984).

[7] G. Lazarides and Q. Shafi, Phys. Lett. B148, 35 (1984).

[8] S. R. Coleman and E. Weinberg, Phys. Rev. D7, 1888 (1973).

[9] A. Albrecht and R. H. Brandenberger, Phys. Rev. D31, 1225 (1985); A. Albrecht, R. H. Brandenberger, and R. Matzner, Phys. Rev. D32, 1280 (1985). 
[10] A. D. Linde, Particle physics and inflationary cosmology (Harwood Academic, 1990), hepth/0503203.

[11] A. D. Linde, Phys. Lett. B129, 177 (1983).

[12] A. R. Liddle and D. H. Lyth, Phys. Lett. B 291, 391 (1992), astro-ph/9208007; A. R. Liddle and D. H. Lyth, Phys. Rept. 231, 1 (1993), astro-ph/9303019.

[13] D. S. Salopek and J. R. Bond, Phys. Rev. D42, 3936 (1990).

[14] E. D. Stewart and D. H. Lyth, Phys. Lett. B302, 171 (1993), gr-qc/9302019.

[15] S. Dodelson and L. Hui, Phys. Rev. Lett. 91, 131301 (2003), astro-ph/0305113; A. R. Liddle and S. M. Leach, Phys. Rev. D68, 103503 (2003) astro-ph/0305263.

[16] D. H. Lyth and A. Riotto, Phys. Rept. 314, 1 (1999), hep-ph/9807278; A. R. Liddle and D. H. Lyth, Cosmological inflation and large-scale structure (Cambridge, UK: Univ. Pr., 2000).

[17] L. Knox and M. S. Turner, Phys. Rev. Lett. 70, 371 (1993), astro-ph/9209006.

[18] H. Georgi and S. L. Glashow, Phys. Rev. Lett. 32, 438 (1974).

[19] I. Dorsner, P. F. Perez, and R. Gonzalez Felipe (2005), hep-ph/0512068.

[20] S. Eidelman et al. [Particle Data Group], Phys. Lett. B592, 1 (2004).

[21] I. Dorsner, private communication.

[22] J. C. Pati and A. Salam, Phys. Rev. D10, 275 (1974).

[23] S. Rajpoot, Phys. Rev. D22, 2244 (1980); G. Lazarides, Q. Shafi and C. Wetterich, Nucl. Phys. B181, 287 (1981); D.-G. Lee, R. N. Mohapatra, M. K. Parida, and M. Rani, Phys. Rev. D51, 229 (1995), hep-ph/9404238; F. Buccella and D. Falcone, Mod. Phys. Lett. A18, 1819 (2003), hep-ph/0304143.

[24] G. Lazarides, M. Magg, and Q. Shafi, Phys. Lett. B97, 87 (1980).

[25] M. Fukugita and T. Yanagida, Phys. Lett. B174, 45 (1986); W. Buchmuller, P. Di Bari, and M. Plumacher, Nucl. Phys. B665, 445 (2003), hep-ph/0302092; G. F. Giudice, A. Notari, M. Raidal, A. Riotto, and A. Strumia, Nucl. Phys. B685, 89 (2004), hep-ph/0310123.

[26] G. Lazarides and Q. Shafi, Phys. Lett. B258, 305 (1991).

[27] V. N. Şenoğuz and Q. Shafi, Phys. Lett. B596, 8 (2004), hep-ph/0403294. 